nab-paclitaxel in patients with resectable disease. Although the primary study endpoint was to determine the effects of this novel chemotherapy regimen on tumour stroma (including an assessment of collagen content and structure of 'cancer-associated fibroblasts' (CAF)), some important lessons can be learned from this innovative study regarding the approach of neoadjuvant chemotherapy in localised pancreatic cancer (Heinemann et al, 2013).

The investigators enroled 16 patients with histologically or cytologically confirmed resectable or borderline resectable exocrine pancreatic cancer that had received two cycles of gemcitabine/nab-paclitaxel. After chemotherapy, 3 patients had progressive disease and 12 patients were able to proceed to surgical resection. Of note, in the final pathological assessment of the surgical specimen, two study patients in fact had a neuroendorcine cancer and not pancreatic adenocarcinoma. No objective response by RECIST was observed after neoadjuvant chemotherapy; however, half of patients had a CA $19-9$ decrease of $>75 \%$ and a partial metabolic response by FDG-PET imaging. The authors reported a median CA 19-9 pre-treatment value of $2.588 \mathrm{U} / \mathrm{dl}$ in their patient population, ranging from 1 to $36.376 \mathrm{U} / \mathrm{dl}$; this would correspond to a median CA 19 9 level of $26 \mathrm{U} \mathrm{ml}^{-1}$ at baseline with a range from $0.01 \mathrm{U} \mathrm{ml}^{-1}$ to 364 $\mathrm{U} \mathrm{ml}^{-1}$, respectively (please note: $(\mathrm{U} / \mathrm{dl})$ is a very uncommon unit for reporting CA 19-9; the most commonly used unit in the literature is $\left(\mathrm{U} \mathrm{ml}^{-1}\right)$ ) (Boeck et al, 2006). On the basis of the evidence from previous CA 19-9 biomarker studies, significantly elevated CA 19-9 levels before surgical resection may indicate a potential subradiographic systemic disease rather than a localised disease. In a single-centre trial from the USA, 51 out of 262 patients who underwent staging laparoscopy for radiographically resectable pancreatic adenocarcinoma in fact had unresectable disease. In this study, the median pre-operative CA 19-9 value for patients who underwent resection was $131 \mathrm{Uml}^{-1}$ vs $379 \mathrm{U} \mathrm{ml}^{-1}$ for those patients with unresectable disease $(P=0.003)$. With the use of a receiver operating characteristics curve for pre-operative CA 19-9 value and tumour resectability, the statistically optimal cut-off value was determined to be $130 \mathrm{U} \mathrm{ml}^{-1}$ (Maithel et al, 2008). In concordance, Katz et al (2010) suggested a pre-treatment CA 19-9 cut-off of 149 $\mathrm{U} \mathrm{ml}^{-1}$ as a predictor of completing chemoradiation and subsequent pancreaticoduodenectomy in their neoadjuvant study (Ketz et al, 2010). In the light of the fact that '.. the content of stroma and CAF may change with different cancer stages' (as the authors themselves stated correctly in the Discussion section), one should keep in mind that an appropriate selection of patients with a unique stage of disease thus should be an important issue in novel translational studies.

Nevertheless, the data presented are important and highlight an innovative approach to how future translational research should be conducted in pancreatic cancer. However, from a clinical point of view, this small study clearly unbosoms potential pitfalls of neoadjuvant treatment approaches in resectable pancreatic cancer: (1) the difficulty of adequately select patients with a real localised disease, (2) the limitations of obtaining an adequate pre-treatment histological diagnosis and (3) the often missing objective response by RECIST to neoadjuvant chemotherapy. The latter one was already described previously in borderline resectable patients and in fact it may be more appropriate to investigate different clinical methods than conventional CT scan for assessing treatment response to a neoadjuvant treatment regimen (e.g. metabolic response by PET or biochemical response by CA 19-9) (Takahashi et al, 2010; Katz et al, 2012).

We thus would recommend including a rigorous histological (no cytological) confirmation of disease before study entry, an assessment of response by different methods other than CT scan and also a CA 19-9 cut-off for patients entering neoadjuvant trials. Such an approach has recently been included in several large phase III adjuvant trials (e.g. RTOG 0848, Prodige 24/Accord 24), mainly by using a post-resection CA 19-9 level of $<180 \mathrm{U} \mathrm{ml}^{-1}$ as an inclusion criterion.

\section{REFERENCES}

Alvarez R, Musteanu M, Garcia-Garcia E, Lopez-Casas PP, Megias D, Guerra C, Muñoz M, Quijano Y, Cubillo A, Rodriguez-Pascual J, Plaza C, de Vicente E, Prados S, Tabernero S, Barbacid M, Lopez-Rios F, Hidalgo M (2013) Stromal disrupting effects of nab-paclitaxel in pancreatic cancer. Br J Cancer 109: 926-933.

Boeck S, Stieber P, Holdenrieder S, Wilkowski R, Heinemann V (2006) Prognostic and therapeutic significance of carbohydrate antigen 19-9 as tumor marker in patients with pancreatic cancer. Oncology 70: 255-264.

Heinemann V, Haas M, Boeck S (2013) Neoadjuvant treatment of borderline resectable and non-resectable pancreatic cancer. Ann Oncol 24: 2484-2492.

Katz MH, Fleming JB, Bhosale P, Varadhachary G, Lee JE, Wolff R, Wang H, Abbruzzese J, Pisters PW, Vauthey JN, Charnsangavej C, Tamm E,

Crane CH, Balachandran A (2012) Response of borderline resectable pancreatic cancer to neoadjuvant therapy is not reflected by radiographic indicators. Cancer 118: 5749-5756.

Katz MH, Varadhachary GR, Fleming JB, Wolff RA, Lee JE, Pisters PW, Vauthey JN, Abdalla EK, Sun CC, Wang H, Crane CH, Lee JH, Tamm EP, Abbruzzese JL, Evans DB (2010) Serum CA 19-9 as a marker of resectability and survival in patients with potentially resectable pancreatic cancer treated with neoadjuvant chemoradiation. Ann Surg Oncol 17: 1794-1801.

Maithel SK, Maloney S, Winston C, Gönen M, D’Angelica MI, Dematteo RP, Jarnagin WR, Brennan MF, Allen PJ (2008) Preoperative CA 19-9 and the yield of staging laparoscopy in patients with radiographically resectable pancreatic adenocarcinoma. Ann Surg Oncol 15: 3512-3520.

Takahashi H, Ohigashi H, Ishikawa O, Eguchi H, Gotoh K, Yamada T, Nakaizumi A, Uehara H, Tomita Y, Nishiyama K, Yano M (2010) Serum CA19-9 alterations during preoperative gemcitabine-based chemoradiation therapy for resectable invasive ductal carcinoma of the pancreas as an indicator for therapeutic selection and survival. Ann Surg 251: 461-469.

\title{
Stellate cells, a point of light in the dark night of pancreatic cancer
}

\section{N Ramírez ${ }^{*}, 1$, A Viúdez ${ }^{2}$, I Hernández-García ${ }^{*}, 2$, D Guerrero ${ }^{3}$, M Gómez-Dorronsoro ${ }^{4}$, F J Herrera ${ }^{5}$, J Vila ${ }^{6}$, L Beloki ${ }^{1}$, M Ciaúrriz $^{1}$, C Mansilla ${ }^{1}$ and R Vera ${ }^{2}$}

\begin{abstract}
${ }^{1}$ Oncohematology Research Group, Navarrabiomed-Miguel Servet Foundation, Pamplona, Spain; ${ }^{2}$ Department of Oncology, Complejo Hospitalario de Navarra, 31008 Pamplona, Spain; ${ }^{3}$ Cancer Epigenetics Group, Navarrabiomed-Miguel Servet Foundation, Pamplona, Spain; ${ }^{4}$ Department of Pathology, Complejo Hospitalario de Navarra, Pamplona, Spain; ${ }^{5}$ Department of Surgery, Complejo Hospitalario de Navarra, Pamplona, Spain and ${ }^{6}$ Endoscopy Unit, Gastroenterology Department, Complejo Hospitalario de Navarra, Pamplona, Spain
\end{abstract}

Sir,

We have read with particular attention the recent article published in the BJC by Alvarez et al (2013).

They, through endoscopic ultrasound elastography system and conventional immuno-histochemical assays, perform an intrinsic characterisation of tumour stroma's stiffness, fibroblast density and architecture focusing in type I collagen fibre arrangement, cancer-associated fibroblasts (CAFs) and activated CAF quantification. The analysis of post-treatment tissues from patients treated with nab-paclitaxel and gemcitabine combination showed a decrease in CAF number and changes in collagen architecture.
These results reaffirm the potential ability of nab-paclitaxel to target the stroma and to change its phenotype, altering the 'hard of the rock', as described by Garber (2010), intensifying its permeability to deliver cytotoxic agents such as gemcitabine by increasing tumour vascularisation or enzymatic inhibition (Frese et al, 2012) that would suppose a better pharmacokinetic profile (Von Hoff et al, 2011). Even though it is known the clinical benefit of nab-paclitaxel plus gemcitabine in metastatic pancreatic cancer, the cellular or molecular mechanisms that are expressly addressing this stromal involution have not been appointed yet (Alvarez et al, 2013). In this sense, authors analysed the role of secreted protein, acidic and rich in 
cysteine (SPARC) although they could not find the correlation between the degree of SPARC expression and clinical or pathological responses due to its high affinity to albumin protein of nab-paclitaxel. SPARC strengthens the accumulation of nab-paclitaxel mediated by albumin in the 'tumour's Achilles' heel' (Von Hoff, Annual meeting of the American Society of Clinical Oncology, 2009); however, the mere use of this endogenous transport system would not justify the clinical impact of this combined treatment. From our point of view the key may be in what Omary et al (2007) described as 'a star on the rise' in pancreatic disease: the pancreatic stellate cells (PaSCs; $\alpha$-SMA + Nestin ++ Vimentin ++ ) because of their role as the main manufacturers of profibrotic extracellular matrix (ECM) components of the pancreatic tumour stroma, and which should match with the activated CAFs (SMA + Vimentin + fibroblasts) in Alvareźs paper.

In pancreatic cancer, PaSCs show increased proliferation and migration properties, and so they could be a suitable target for nab-paclitaxel because of their ability to interfere with the mitotic activity (Gradishar, 2006). Alvarez et al (2013) demonstrate that although CAFs number decreases in patients treated with nab-paclitaxel plus gemcitabine in neoadjuvant setting, the average of activated CAFs remains unchanged.

We would like to explain our hypothesis related to what is happening in the stroma: it could be a transient blockade of activated CAFs metabolism, a quiescent status forced for a pharmacologically active substance. Bachem et al (2005) show that cancer cells (CCs) induce a desmoplastic reaction in pancreatic adenocarcinoma by stimulating PaSCs in a paracrine way. So it could be an indirect elimination of principal fibrogenic mediators that stimulate proliferation (platelet-derived growth factor) and ECM synthesis (fibroblast growth factor -2 and transforming growth factor $-\beta 1$ ) of activated PaSCs through the abrogation of CCs. In this sense, the hypothetical presence of surface cellular receptors for nab-paclitaxel in CCs could be an interesting pathway biomarker for the effectiveness of the drug as authors related. On the other hand, the ablation of physiological PaSCs functioning after nab-paclitaxel inclusion, in turn, would break the two-way communication between PaSCs and CCs (Apte et al, 2013). In this context, under normal conditions CCs recruit new PaSCs to their vicinity (Vonlaufen et al, 2008), whereas nab-paclitaxel would temporarily inhibit the main mechanism that rules the desmoplastic reaction.

All these molecular mechanisms would allow to maintain and stabilise the activated CAF's number despite them being dysfunctional, and also would decline its CAF progeny. In this sense, Apte et al (2013) and Bachem et al (2005) propose that activated PaSCs can transform into a myofibroblast-like phenotype sub-population with the ability of secreting excess amounts of ECM.

In conclusion, due to the dynamic nature of the stromal compartment, it is critically involved in the development and progresion of pancreatic tumours (Heinemann et al, 2013). Before using neadjuvant treatment it may be important to know the stroma's cellular activation grade with regard to PaSCs plus their number. In this sense, PaSCs or activated CAFs could give us ample information about tumoral potential of the stroma itself and so could have an important contribution for the patient's prognosis. Also, the fact that activated CAFs do not decrease after nab-paclitaxel treatment could not mean that these cells are not one of the main actors of stromal disruption but the primary target of nab-paclitaxel.

\section{ACKNOWLEDGEMENTS}

LB is a recipient of APPICS predoctoral fellowship from the Departamento de Salud del Gobierno de Navarra. MC is a recipient of PFIS predoctoral fellowship from ISCIII. CM is a recipient of ANABASID from the Departamento de Educación del Gobierno de Navarra.

\section{CONFLICT OF INTEREST}

The authors declare no conflict of interest.

\section{REFERENCES}

Alvarez R, Musteanu M, Garcia-Garcia E, Lopez-Casas PP, Megias D, Guerra C, Muñoz M, Quijano Y, Cubillo A, Rodriguez-Pascual J, Plaza C, de Vicente E, Prados S, Tabernero S, Barbacid M, Lopez-Rios F, Hidalgo M (2013) Stromal disrupting effects of nab-paclitaxel in pancreatic cancer. Br J Cancer 109(4): 926-933.

Apte MV, Wilson JS, Lugea A, Pandol SJ (2013) A starring role for stellate cells in the pancreatic cancer microenvironment. Gastroenterology 144: 1210-1219.

Bachem MG, Schunemann M, Ramadani M, Siech M, Beger H, Buck A, Zhou S, Schmid-Kotsas A, Adler G (2005) Pancreatic carcinoma cells induce fibrosis by stimulating proliferation and matrix synthesis of stellate cells. Gastroenterology 128: 907-921.

Frese KK, Neesse A, Cook N, Bapiro TE, Lolkema MP, Jodrell DI, Tuveson DA (2012) nab-Paclitaxel potentiates gemcitabine activity by reducing cytidine deaminase levels in a mouse model of pancreatic cancer. Cancer Discov 2: 260-269.

Garber K (2010) Stromal depletion goes on trial in pancreatic cancer. J Natl Cancer Inst 102: 448-450.

Gradishar WJ (2006) Albumin-bound paclitaxel: a next-generation taxane. Expert Opin Pharmacother 7: 1041-1053.

Heinemann V, Reni M, Ychou M, Richel DJ, Macarulla T, Ducreux M (2013) Tumour-stroma interactions in pancreatic ductal adenocarcinoma: Rationale and current evidence for new therapeutic strategies. Cancer Treat Rev 40(1): $118-128$.

Omary MB, Lugea A, Lowe AW, Pandol SJ (2007) The pancreatic stellate cell: a star on the rise in pancreatic diseases. J Clin Invest 117: 50-59.

Von Hoff DD, Ramanathan RK, Borad MJ, Laheru DA, Smith LS, Wood TE, Korn RL, Desai N, Trieu V, Iglesias JL, Zhang H, Soon-Shiong P, Shi T, Rajeshkumar NV, Maitra A, Hidalgo M (2011) Gemcitabine plus nab-paclitaxel is an active regimen in patients with advanced pancreatic cancer: a phase I/II trial. J Clin Oncol 29: 4548-4554.

Vonlaufen A, Phillips PA, Xu Z, Goldstein D, Pirola RC, Wilson JS, Apte MV (2008) Pancreatic stellate cells and pancreatic cancer cells: an unholy alliance. Cancer Res 68: $7707-7710$.

*Correspondence: Dr I Hernández-García; E-mail: irene.hernandez.garcia@cfnavarra.es or Dr N Ramírez; E-mail: natalia.ramirez.huerto@cfnavarra.es

\title{
Reply: 'Comments on Stromal disrupting effects of nab-paclitaxel in pancreatic cancer'
}

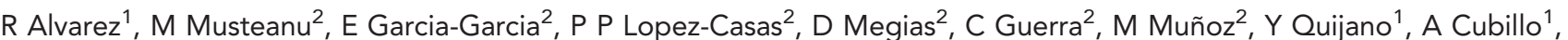

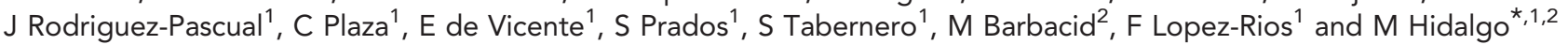

${ }^{1}$ Centro Integral Oncológico Clara Campal (CIOCC), Oña 10, 28050 Madrid, Spain and ${ }^{2}$ Spanish National Cancer Research Centre (CNIO), Melchor Fernández Almagro 3, 28029 Madrid, Spain

Sir,

In response to Boeck et al

We read with interest the comments made by Boeck et al (2014) about our study. We appreciate their attention to the units used to report CA19.9 levels, which indeed should have been $\mathrm{U} \mathrm{ml}^{-1}$ and not $\mathrm{U} \mathrm{dl}^{-1}$ as stated. We certainly agree that a high level of CA19.9 at diagnosis may be an indication of advanced disease and that this should be considered in the selection criteria in preoperative studies. Indeed in our study, with small sample size, one patient with very high CA19.9 level who actually progressed during chemotherapy skewed that average level of CA19.9. This patient was not operated and therefore does not affect the tissue results. As Boeck et al mention, levels of CA19.9 should be either a selection criteria or a stratification factor in outcome-oriented preoperative studies that should also include better imaging methods to determine responses and histological, rather than cytological, diagnosis. In our study, however, as the goals were to determine the effects of Nab-paclitaxel in tumour tissue, this criterion was not part of the eligibility criteria. We agree, however, that future controlled studies to confirm our observations should exclude patients with elevated CA19.9 and plan to do so.

In clinical practice, however, one of the goals of preoperative treatment is to identify patients with more advanced or resistant disease who can be 